\title{
Continuous weak measurement and feedback control of a solid-state charge qubit: A physical unravelling of non-Lindblad master equation
}

\author{
Shi-Kuan Wang, Jinshuang Jin,* and Xin-Qi $\mathrm{Li}^{\dagger}$ \\ State Key Laboratory for Superlattices and Microstructures, Institute of Semiconductors, \\ Chinese Academy of Sciences, P.O. Box 912, Beijing 100083, China
}

(Received 24 November 2006; revised manuscript received 6 February 2007; published 4 April 2007)

\begin{abstract}
Conventional quantum trajectory theory developed in quantum optics is largely based on the physical unravelling of a Lindblad-type master equation, which constitutes the theoretical basis of continuous quantum measurement and feedback control. In this work, in the context of continuous quantum measurement and feedback control of a solid-state charge qubit, we present a physical unravelling scheme of a non-Lindblad-type master equation. Self-consistency and numerical efficiency are well demonstrated. In particular, the control effect is manifested in the detector noise spectrum, and the effect of measurement voltage is discussed.
\end{abstract}

DOI: 10.1103/PhysRevB.75.155304

PACS number(s): 42.50.Lc, 03.65.Ta, 03.67.- a, 85.35.Be

\section{INTRODUCTION}

Quantum trajectory theory has been developed and intensively applied in quantum optics. ${ }^{1-7}$ However, the theory is largely based on the unravelling of a Lindblad-type master equation, which has clear physical interpretation. ${ }^{6}$ For a nonLindblad-type master equation, ${ }^{8}$ or even the non-Markovian dissipative dynamics, ${ }^{9-12}$ unravelling in terms of a stochastic differential equation has also been established for various purposes. Nevertheless, these schemes lack physical interpretation, thus having only mathematical meaning.

In a solid-state system, the quantum measurement ${ }^{13-17}$ of solid-state qubit and feedback control ${ }^{18-20}$ have been an extensively studied subject in recent years, largely stimulated by the prospect of solid-state quantum computing. The theoretical description of this solid-state qubit measurement problem was developed originally in terms of the $n$-resolved master equation, ${ }^{13,14}$ where $n$ stands for the number of electrons passed through the measurement apparatus. Alternatively, Bayesian formalism ${ }^{15}$ and also the conventional quantum trajectory theory ${ }^{17}$ were developed for this solid-state measurement setup. Being of interest, it can be shown that all these three approaches are precisely equivalent to each other.

More specifically, for the setup of a pair of coupled quantum dot qubit measured by a quantum point contact (QPC) detector, which has become an experimentally studied system, ${ }^{21}$ we found that the above-mentioned theories were restricted to the limit of a large measurement voltage across the QPC. ${ }^{22,23}$ At finite voltages, i.e., as the measurement voltage is comparable to or not much higher than the qubit's intrinsic energy scale, the measurement dynamics is governed by a non-Lindblad-type master equation. ${ }^{22}$ In this regime, the $n$-resolved master equation was also developed to study the readout characteristics. ${ }^{23}$ In the present work, we further extend it to a quantum trajectory theory, which conditions the state evolution on the entire measurement records. It is well known that this kind of description is essential to the quantum feedback control and other possible applications.

The paper is organized as follows. In Sec. II, we first present a brief description for the setup under study, then outline the $n$-resolved master equation for the measurement. In connection with the conditional state evolution, a physical unravelling scheme is constructed and a practical Monte Carlo simulation is carried out. Also, for completeness and latter use, a generalized quantum-jump approach is formulated for the calculation of noise spectrum. For the sake of brevity, some complicated expressions and mathematical details are presented in Appendixes A and B. In Sec. III, we apply the developed formalism to the study of feedback control based on a suboptimal feedback algorithm. Finally, the conclusion is presented in Sec. IV.

\section{CONTINUOUS MEASUREMENT OF A CHARGE QUBIT}

\section{A. Model description}

Let us consider a solid-state charge qubit measured by a nearby mesoscopic detector. In this work, as well studied in literature, ${ }^{13,15-17}$ the charge qubit is modeled by a pair of coherently coupled quantum dots with an extra electron in it, and the mesoscopic detector can be a mesoscopic QPC. The Hamiltonian of this qubit-plus-detector setup reads

$$
\begin{gathered}
H=H_{0}+H^{\prime}, \\
H_{0}=H_{q b}+\sum_{k}\left(\epsilon_{k}^{L} c_{k}^{\dagger} c_{k}+\epsilon_{k}^{R} d_{k}^{\dagger} d_{k}\right), \\
H_{q b}=\epsilon_{a}|a\rangle\left\langle a\left|+\epsilon_{b}\right| b\right\rangle\langle b|+\Omega(|b\rangle\langle a|+| a\rangle\langle b|), \\
H^{\prime}=\sum_{k, q}\left[\left(\mathcal{T}_{k q}+\chi_{k q}|a\rangle\langle a|\right) c_{k}^{\dagger} d_{q}+\text { H.c. }\right] .
\end{gathered}
$$

In this decomposition, the free part of the total Hamiltonian, $H_{0}$, contains Hamiltonians of the measured qubit $H_{\mathrm{qb}}$ and the QPC's reservoirs. $H^{\prime}$ describes the tunnel coupling between the QPC's two reservoirs, with tunneling amplitudes depending on the qubit states, i.e., $\mathcal{T}_{k q}+\chi_{k q}|a\rangle\langle a|$. The operators $c_{k}^{\dagger}$ $\left(c_{k}\right)$ and $d_{k}^{\dagger}\left(d_{k}\right)$ are, respectively, the electronic creation (annihilation) operators of the QPC's left and right reservoirs. $|a\rangle$ and $|b\rangle$ stand for the logic states of the qubit, with coher- 
ent coupling $\Omega$ during manipulation. Introducing $\epsilon=\left(\epsilon_{a}\right.$ $\left.-\epsilon_{b}\right) / 2$ and taking $\left(\epsilon_{a}+\epsilon_{b}\right) / 2$ as the reference energy, the qubit eigenenergies read $E_{1}=\sqrt{\epsilon^{2}+\Omega^{2}}=\Delta / 2$ and $E_{2}$ $=-\sqrt{\epsilon^{2}+\Omega^{2}}=-\Delta / 2$. Correspondingly, the eigenstates are $|1\rangle$ $=\cos \frac{\theta}{2}|a\rangle+\sin \frac{\theta}{2}|b\rangle$ for the excited state and $|0\rangle=\sin \frac{\theta}{2}|a\rangle$ $-\cos \frac{\theta}{2}|b\rangle$ for the ground state, where $\theta$ is defined by $\cos \theta$ $=2 \epsilon / \Delta$ and $\sin \theta=2 \Omega / \Delta$.

For the sake of simplicity, we further assume that the tunneling amplitudes are real and independent of the reservoir electron states, i.e., $\mathcal{T}_{k q}=\mathcal{T}$ and $\chi_{k q}=\chi$. Accordingly, depending on the qubit states $|a\rangle$ and $|b\rangle$, the detector currents read $I_{a}=2 \pi g_{L} g_{R}(\mathcal{T}+\chi)^{2} V$ and $I_{b}=2 \pi g_{L} g_{R} \mathcal{T}^{2} V$, respectively. Here $V$ is the voltage across the tunnel junction of the QPC, and $g_{L(R)}$ are the densities of states of the reservoir electrons. Crucially, $\Delta I=I_{a}-I_{b}$ characterizes the detector's response to the qubit states. In this work, we assume that the detector operates in the so-called weakly responding regime, i.e., $\Delta I$ $\ll I_{0}=\left(I_{a}+I_{b}\right) / 2$. In this regime, the detector's output current can be treated as a continuous diffusive variable.

\section{B. $n$-resolved master equation}

The so-called $n$-resolved master equation is obtained by partially tracing out the detector's microscopic degrees of freedom but keeping track of the number $n$ of electrons that have tunneled through the detector during the time period $(0, t) .{ }^{13,14,23}$ Originally, it was derived in Ref. 13 from the many-body Schrödinger equation at zero temperature and in the large measurement voltage limit. Later, it was proved that this approach is completely equivalent to the Bayesian approach $^{15}$ and the quantum trajectory theory, ${ }^{17}$ which share the same Lindblad-type master equation and its unravelling.

Alternatively, under arbitrary (i.e., not high enough) voltages, it was found that this measurement problem cannot be described by a Lindblad-type master equation. ${ }^{22,23}$ In particular, a non-Lindblad-type master equation was derived, ${ }^{22}$ and its $n$-resolved counterpart reads ${ }^{23}$

$$
\begin{aligned}
\dot{\rho}^{(n)}= & -i \mathcal{L} \rho^{(n)}-\frac{1}{2}\left\{Q \widetilde{Q} \rho^{(n)}-\widetilde{Q}^{(-)} \rho^{(n-1)} Q\right. \\
& \left.-\widetilde{Q}^{(+)} \rho^{(n+1)} Q+\text { H.c. }\right\} .
\end{aligned}
$$

Here, $Q=\mathcal{T}+\chi|a\rangle\langle a|, \quad \widetilde{Q}=\widetilde{Q}^{(+)}+\widetilde{Q}^{(-)}, \quad \widetilde{Q}^{( \pm)}=\widetilde{C}^{( \pm)}(\mathcal{L}) Q$, and $\widetilde{C}^{( \pm)}(\mathcal{L})=\int_{-\infty}^{\infty} d t C^{( \pm)}(t) e^{-i \mathcal{L} t} . C^{( \pm)}(t)$ are the reservoir electron correlation functions. Under wideband approximation for the QPC reservoirs, the spectral functions $\widetilde{C}^{( \pm)}(\mathcal{L})$ can be explicitly carried out as $\widetilde{C}^{( \pm)}(\mathcal{L})=2 \pi g_{L} g_{R}\left[x /\left(1-e^{-x / T}\right)\right]_{x=-\mathcal{L} \mp V}$, where $T$ is the reservoir temperature (in this work, we use the unit system of $\hbar=e=k_{B}=1$ ). It is of interest to note that the Liouvillian operator $\mathcal{L}$ in $\widetilde{C}^{( \pm)}(\mathcal{L})$ contains the information of energy exchange between the detector and the qubit, which correlates the energy relaxation of the measured qubit with the inelastic electron tunneling in the detector. Note also that in the derivation of the above $n$-resolved master equation, we did not make an assumption of a large bias voltage across the QPC detector. At large voltage limit, i.e., the bias voltage is much larger than the internal energy scale of the qubit, the spectral function $\widetilde{C}^{( \pm)}(\mathcal{L}) \simeq \widetilde{C}^{( \pm)}(0)$ and Eq. (2) reduces to the result obtained in Ref. 13.

Formally, we rewrite Eq. (2) as

$$
\dot{\rho}^{(n)}=-i \mathcal{L} \rho^{(n)}-\mathcal{R} \rho^{(n)}+\mathcal{R}_{1} \rho^{(n-1)}+\mathcal{R}_{2} \rho^{(n+1)},
$$

where $\mathcal{R}, \mathcal{R}_{1}$, and $\mathcal{R}_{2}$ are superoperators defined in accordance with Eq. (2). To solve this infinite number of coupled equations, we perform the discrete Fourier transformation, $\rho(k, t)=\Sigma_{n} e^{i n k} \rho^{(n)}(t)$, yielding

$$
\dot{\rho}(k, t)=\left[-i \mathcal{L}-\mathcal{R}+e^{i k} \mathcal{R}_{1}+e^{-i k} \mathcal{R}_{2}\right] \rho(k, t) .
$$

Explicitly, in the localized dot-state representation $\{|a\rangle,|b\rangle\}$, we obtain

$$
\left(\begin{array}{c}
\dot{\rho}_{a a} \\
\dot{\rho}_{b b} \\
\dot{\rho}_{a b} \\
\dot{\rho}_{b a}
\end{array}\right)=\left(\begin{array}{cccc}
a_{1} & 0 & a_{2}+i \Omega & a_{2}-i \Omega \\
0 & b_{1} & b_{2}-i \Omega & b_{2}+i \Omega \\
c_{3}+i \Omega & c_{2}-i \Omega & c_{1}-i \epsilon_{a}+i \epsilon_{b} & 0 \\
c_{3}-i \Omega & c_{2}+i \Omega & 0 & c_{1}+i \epsilon_{a}-i \epsilon_{b}
\end{array}\right)\left(\begin{array}{c}
\rho_{a a} \\
\rho_{b b} \\
\rho_{a b} \\
\rho_{b a}
\end{array}\right)
$$

For brevity, the explicit expressions of the coefficients $a_{1(2)}$, $b_{1(2)}$, and $c_{1(2,3)}$ are ignored here and are put alternatively in Appendix A.

Formally, we reexpress the Fourier-transformed master equation as $\dot{\rho}(k, t)=\mathcal{M}(k) \rho(k, t)$, and the solution reads $\rho(k, t)=e^{\mathcal{M}(k)\left(t-t_{0}\right)} \rho\left(k, t_{0}\right)$. Note that we are concerned with the $n$-resolved state evolution from $t_{0}$ to $t$, i.e., the counting of $n$ starts from the moment $t_{0}$. We thus have $\rho^{(n)}\left(t_{0}\right)$ $=\rho\left(t_{0}\right) \delta_{n, 0}$ and $\rho\left(k, t_{0}\right)=\rho\left(t_{0}\right)$. With the knowledge of $\rho(k, t)$, the inverse Fourier transform gives

$$
\rho^{(n)}(t)=\sum_{k} e^{-i n k} \rho(k, t)=\sum_{k} e^{-i n k} e^{\mathcal{M}(k)\left(t-t_{0}\right)} \rho\left(t_{0}\right) .
$$

Strikingly, we can introduce a propagator for the state evolution, $\mathcal{U}(n, t)=\sum_{k} e^{-i n k} e^{\mathcal{M}(k) t}$. Since this propagator is completely determined by the dynamic structure of the master equation but does not depend on the initial state, we can 
numerically evaluate it by a "one-time task" such as the fast Fourier transformation. This feature leads to a very efficient Monte Carlo simulation for the measurement-history conditioned evolution (i.e., the quantum trajectory simulation).

\section{Monte Carlo simulation for conditional evolution}

It seems that $\rho^{(n)}(t)$ contains less information about the measurement record (history) than the conditional state $\rho_{c}(t)$ in the conventional quantum trajectory theory, ${ }^{17}$ since it only implies that totally there have been $n$ electrons passed through the QPC junction during the specified time period. However, if we make successive readout for the electron numbers $n_{k}$ passed through the detector during the time interval $\left(t_{k-1}, t_{k}\right)$, we actually record the measurement current $I_{c}(t)$ of a single realization. After every read out of $n_{k}$, the statistically mixed state $\rho^{(n)}\left(t_{k}\right)$ with any possible $n$ would "collapse" to a normalized state $\rho^{\left(n_{k}\right)}\left(t_{k}\right)$ with definite $n=n_{k}$. The set of records $\left\{n_{k}: k=1,2, \ldots\right\}$ corresponds to the current $I_{c}(t)$, and the set of states $\left\{\rho^{\left(n_{k}\right)}\left(t_{k}\right): k=1,2, \ldots\right\}$ is nothing but the conditional state $\rho_{c}(t)$ in the quantum trajectory theory.

This state-update procedure based on the $n$-resolved master equation was introduced in Ref. 15, where its exact equivalence to the Bayesian and quantum trajectory theories was analytically proved. That is, the conditional master equation can be rederived based on the $n$-resolved master equation together with the above collapse idea. However, we found that this can be done only in the large voltage limit, which leads to a Lindblad-type master equation. ${ }^{15,17}$ For arbitrary voltage, rather than deriving a conditional master equation to describe the measurement-record conditioned evolution, here we would like to develop an efficient numerical unravelling scheme which has the advantage of being applicable to a non-Lindblad-type master equation as studied in this work.

More quantitatively, let us consider the state evolution during $\left[t_{j}, t_{j}+\tau\right]$. That is, starting with a definite state at $t_{j}$, say $\rho\left(t_{j}\right)$, the state $\rho^{\left(n_{j}\right)}\left(t_{j}+\tau\right)$ at $t_{j}+\tau$ can be calculated via

$$
\rho^{\left(n_{j}\right)}\left(t_{j}+\tau\right)=\mathcal{U}\left(n_{j}, \tau\right) \rho\left(t_{j}\right) .
$$

If the measurement is made but the result is ignored, the (mixture) state is described by

$$
\rho\left(t_{j}+\tau\right)=\sum_{n_{j}} \rho^{\left(n_{j}\right)}\left(t_{j}+\tau\right)=\sum_{n_{j}} \operatorname{Pr}\left(n_{j}\right) \rho_{c}\left(n_{j}, t_{j}+\tau\right),
$$

where $\operatorname{Pr}\left(n_{j}\right)=\operatorname{Tr}\left[\rho^{\left(n_{j}\right)}\left(t_{j}+\tau\right)\right]$ stands for the probability of having $n_{j}$ electrons tunneled through the detector, and $\rho_{c}\left(n_{j}, t_{j}+\tau\right)=\rho^{\left(n_{j}\right)}\left(t_{j}+\tau\right) / \operatorname{Pr}\left(n_{j}\right)$ is the normalized state conditioned by the definite number of $n_{j}$ electrons observed to have passed through the detector.

The second equality of Eq. (8) implies that if we stochastically generate $n_{j}$ according to the probability $\operatorname{Pr}\left(n_{j}\right)$ for each time interval $\left[t_{j}, t_{j}+\tau\right]$, step by step from $t_{0}$ to $t$, and collapse the state definitely onto $\rho_{c}\left(n_{j}, t_{j}+\tau\right)$, i.e., $\rho_{c}\left(t_{j}+\tau\right)$ $=\rho^{\left(n_{j}\right)}\left(t_{j}+\tau\right) / \operatorname{Pr}\left(n_{j}\right)=\mathcal{U}\left(n_{j}, \tau\right) \rho\left(t_{j}\right) / \operatorname{Tr}\left[\mathcal{U}\left(n_{j}, \tau\right) \rho\left(t_{j}\right)\right]$, we have in fact simulated a particular realization for the selective state evolution conditioned on the (continuous) specific measurement results. The simple ensemble average over a large

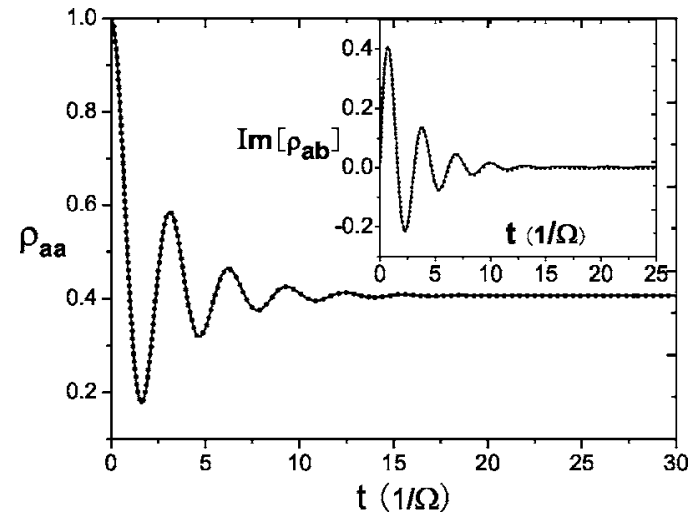

FIG. 1. Ensemble average of the Monte Carlo simulation over 500 quantum trajectories (dotted line) versus the result directly given by the unconditional master equation (solid line). It is assumed that the initial state of the qubit $|\psi\rangle=|a\rangle$. The relevant parameters are $\mathcal{T}=20 \Omega, \chi=0.7 \Omega, V=0.5 \Omega, \epsilon=0.25 \Omega, T=1.0 \Omega$, and $g_{L(R)}=1 / \sqrt{2 \pi} \Omega$.

number of particular realizations of $\rho_{c}(t)$ recovers the unconditional state $\rho(t)$. Obviously, this unravelling scheme is completely equivalent to the spirit of the conventional quantum trajectory theory, despite that in this context we are unable to derive an explicit stochastic differential equation to unravel the underlying non-Lindblad master equation. However, they have precisely the same physical meaning.

To stochastically generate $n_{j}$ according to the probability $\operatorname{Pr}\left(n_{j}\right)$, two procedures are adopted as follows: (i) Based on the ensemble average current $I(t)=\operatorname{Re}\{\operatorname{Tr}[\bar{Q} \rho(t) Q]\}$, which was derived in Ref. 23, the output current in a particular measurement realization reads

$$
I_{c}(t)=\operatorname{Re}\left\{\operatorname{Tr}\left[\bar{Q} \rho_{c}(t) Q\right]\right\}+\xi(t) .
$$

The first term in this equation is related to the conditional evolution of qubit. The second noisy term $\xi(t)$ originates from the detector's intrinsic noise, which is a Poisonian variable in the regime of point process and a Gaussian variable in the diffusive regime. In the latter case, $\xi(t)$ has zero mean value and the spectral density $S_{\xi}=2 I_{0} \operatorname{coth} \frac{V}{2 T}$. At zero temperature and large voltage limit, this treatment recovers the existing result of quantum trajectory theory and Bayesian approach, ${ }^{15,17}$ i.e., $I_{c}(t)=\rho_{c, a a}(t) I_{a}+\rho_{c, b b}(t) I_{b}+\xi(t)$, with $S_{\xi}=2 I_{0}$. (ii) Straightforwardly, in our simulation we relate the stochastic electron number $n_{j}$ with $I_{c}(t) \quad$ via $\quad n_{j}=\int_{t_{j}}^{t_{j}+\tau} d t^{\prime} I_{c}\left(t^{\prime}\right)=\bar{I}_{c}\left(t_{j}\right) \tau+d W\left(t_{j}\right)$, where $\bar{I}_{c}\left(t_{j}\right)$ $=\operatorname{Re}\left\{\operatorname{Tr}\left[\bar{Q} \rho_{c}\left(t_{j}\right) Q\right]\right\}$ and $d W\left(t_{j}\right)$ is the Wiener increment during $\left[t_{j}, t_{j}+\tau\right]$.

In Fig. 1 we plot a comparison of the ensemble average of the Monte Carlo simulation (over 500 quantum trajectories) with the result directly given by the unconditional master equation. The excellent agreement shows the validity and efficiency of the proposed unravelling scheme.

In this context, two points are likely to be highlighted: (i) the measurement voltage considered here is moderately finite, but not the high voltage limit; ${ }^{15,17}$ (ii) the corresponding non-Lindblad master equation is unravelled physically, hav- 


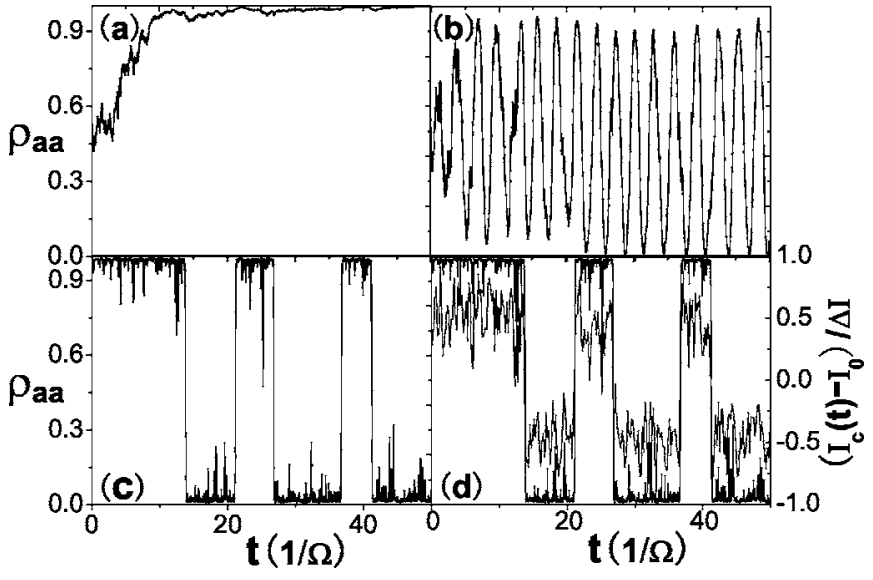

FIG. 2. The main characteristics of the conditional state evolution under continuous measurement: (a) gradual localization $(\Omega$ =0) from an initial superposition state $|\psi\rangle=1 / \sqrt{2}(|a\rangle+|b\rangle)$, (b) gradual purification from a completely mixed state, (c) Zeno effect under relatively strong continuous measurement, and (d) conditional state evolution (thick line) versus the filtered output current (thin line), which is obtained by $\frac{1}{\Delta t} \int_{t}^{t+\Delta t} I_{c}\left(t^{\prime}\right) d t^{\prime}$, with the filtering window $\Delta t=0.2 / \Omega$. The parameters $(\mathcal{T}, \chi, V, \epsilon$, and $T)$ are adopted as follows (in units of $\Omega$ ): (a) $(20.0,0.13,3.0,0.25$, and 1.0), (b) (20.0, 0.7, 3.0, 0.25, and 1.0), (c) (25.0, 4.0, 3.0, 0.25, and 1.0), and (d) $\left(25.0,4.0,3.0,0.25\right.$, and 1.0). The density of states $g_{L(R)}$ $=1 / \sqrt{2 \pi} \Omega$.

ing the same physical interpretation as provided by Wiseman and Milburn. ${ }^{6}$

In Fig. 2 we show the main features of the conditional state evolution. ${ }^{15,24}$ (i) Assuming that the coherent coupling is switched off $(\Omega=0)$, in Fig. 2(a) we illustrate the wavefunction collapse of a pure state $|\psi\rangle=1 / \sqrt{2}|a\rangle+|b\rangle$ under measurement. This feature has great implication in understanding the measurement postulate in quantum mechanics, which has been highlighted by the concept of gradual collapse for a typical solid-state two-level state under (weak) measurement, as discussed in particular by Caldeira and Leggett. $^{25}$ The reason for this gradual collapse is the weak coupling and the finite noise of the detector, which make the quantum measurement need some time until acceptable signal-to-noise ratio is reached. (ii) On the other hand, if $\Omega$ $\neq 0$, the ideal measurement will lead to the gradual purification of the qubit state starting, for instance, with a completely mixed state. This is shown by the revival of the coherent oscillation in Fig. 2(b). It is well known that for strong projective measurement the qubit will collapse to a definite eigenstate of the observable. In the present context, it is " $\sigma_{z}$," whose eigenstates are the qubit states $|a\rangle$ and $|b\rangle$. In contrast, the continuous weak measurement, which is our concern here, only partially collapses the qubit state after each relatively short time measurement. After each partial collapse, the purity of the qubit state will increase. As a consequence, the continuous weak measurement, which is equivalent to a large number of repeated short-time measurements along time, will lead to the gradual purification shown in Fig. 2(b) (iii) As shown in Fig. 2(c), with the increase of the measurement strength $(|\chi| / \Omega)$, the duration time on each qubit state is enhanced, while the switching time between them is re- duced. This is an obvious signature of the quantum Zeno effect, appearing in the regime of gradual but not the conventional instantaneous collapse. (iv) In the conditional dynamics, the output current $I_{c}(t)$ can basically follow the conditional qubit state, as shown by Fig. 2(d). Due to the intrinsic noise of the detector, here the filtered current, i.e., $\bar{I}_{c}(t)=\frac{1}{\Delta t} \int_{t}^{t+\Delta t} I_{c}\left(t^{\prime}\right) d t^{\prime}$, is plotted, where $\Delta t$ is the "filtering window."

\section{Noise spectrum}

In the same spirit of conventional quantum trajectory theory, ${ }^{6,17}$ the present unravelling scheme also provides a natural way of calculating the output power spectrum. The details of the derivation is referred to Appendix B; here we simply present the resultant expression of the output current correlator, which reads

$$
\begin{aligned}
K_{I}(\tau) \equiv & E\left[I_{c}(t+\tau) I_{c}(t)\right]-E\left[I_{c}(t+\tau)\right] E\left[I_{c}(t)\right] \\
= & \operatorname{Tr}\left[\overline{\mathcal{U}} e^{\mathcal{L} \tau} \overline{\mathcal{U}} \rho(t)\right]-\operatorname{Tr}[\overline{\mathcal{U}} \rho(t+\tau)] \operatorname{Tr}[\overline{\mathcal{U}} \rho(t)] \\
& +\operatorname{Tr}\left[\overline{\mathcal{U}}^{\prime} \rho(t)\right] \delta(\tau),
\end{aligned}
$$

where $\overline{\mathcal{U}}=\Sigma_{n} n \mathcal{U}(n, d t) / d t, \overline{\mathcal{U}}^{\prime}=\Sigma_{n} n^{2} \mathcal{U}(n, d t) / d t$, and $\rho(t+\tau)$ $=e^{\mathcal{L} \tau} \rho(t)$. For $\quad$ stationary state, $\quad K_{I}(\tau)=\operatorname{Tr}\left[\overline{\mathcal{U}} e^{\mathcal{L}} \overline{\mathcal{U}} \rho(\infty)\right]$ $-\operatorname{Tr}[\overline{\mathcal{U}} \rho(\infty)]^{2}+\operatorname{Tr}\left[\overline{\mathcal{U}}^{\prime} \rho(\infty)\right] \delta(\tau)$.

In practice, particularly in the presence of quantum feedback, the ensemble averaged evolution represented by $e^{\mathcal{L} \tau}$ can be implemented by numerically averaging the stochastic trajectories. In the absence of quantum feedback, the above quantum trajectory approach can precisely recover the analytic result of the stationary-state noise spectrum, obtained by using the MacDonald formula. ${ }^{23}$

\section{QUANTUM FEEDBACK CONTROL}

Quantum feedback control is one of the typical means of quantum coherence control. In quantum optics, the study of quantum feedback control has been going on for more than a decade. ${ }^{26}$ Elegant applications of the real-time feedback technique may be exemplified by the adaptive phase measurement with the ability of reaching the quantum limits, ${ }^{27}$ as well as the scalable quantum computation. ${ }^{28}$ However, it is a relatively new subject in solid states. ${ }^{18-20}$ In particular, the conditional state evolution under continuous weak measurement has been experimentally demonstrated in solid-state qubit very recently. ${ }^{29}$ This may pave a way to the quantum feedback control in solid states. For the solid-state setup under the present study, we now consider the feedback control of the qubit coherent evolution by unravelling the underlying measurement dynamics that is, in general, governed by a non-Lindblad master equation.

The basic idea is to convert the measurement information in the output current $I_{c}(t)$ into the evolution of a qubit state $\rho_{c}(t)$. By comparing $\rho_{c}(t)$ with the desired state $\rho_{d}(t)$, their difference is then employed to modify the qubit Hamiltonian in order to reduce their difference in the next evolution step. Specifically, we consider a symmetric qubit (i.e., $\epsilon=0)$. The 
desired state is $\left|\psi_{d}(t)\right\rangle=\cos (\Omega t)|a\rangle-i \sin (\Omega t)|b\rangle$. In real-time feedback control, each successive feedback acts only for an infinitesimal time interval $\Delta t$. In the so-called Bayesian stateestimate-based feedback, ${ }^{30}$ the suboptimal algorithm is desirable. ${ }^{31,32}$ That is, the algorithm is constructed such that the state evolution in each infinitesimal time step will maximize the fidelity of the estimated state with the desired (target) state. In more details, as far as the term related to the feedback Hamiltonian is concerned, the state $\rho_{c}(t+\Delta t)$ is given by

$$
\begin{aligned}
\rho_{c}(t+\Delta t)= & \rho_{c}(t)-i\left[H_{f b}, \rho_{c}(t)\right] \Delta t-\frac{1}{2}\left[H_{f b},\left[H_{f b}, \rho_{c}(t)\right]\right](\Delta t)^{2} \\
& +\ldots
\end{aligned}
$$

The fidelity of this state with the target state reads

$$
\begin{aligned}
\left\langle\psi_{d}(t)\right| & \rho_{c}(t+\Delta t)\left|\psi_{d}(t)\right\rangle \\
= & \left\langle\psi_{d}(t)\left|\rho_{c}(t)\right| \psi_{d}(t)\right\rangle-i\left\langle\psi_{d}(t)\left|\left[H_{f b}, \rho_{c}(t)\right]\right| \psi_{d}(t)\right\rangle \Delta t \\
& -\frac{1}{2}\left\langle\psi_{d}(t)\left|\left[H_{f b},\left[H_{f b}, \rho_{c}(t)\right]\right]\right| \psi_{d}(t)\right\rangle(\Delta t)^{2}+\ldots
\end{aligned}
$$

To optimize the fidelity, one should maximize the coefficient of $\Delta t$, which is the dominant term. Similar to other control theories, the maximization must be subject to certain constraints, e.g., the restriction on the maximum eigenvalue of $H_{f b}$, the sum of the norms of the eigenvalues, or the sum of the squares of the eigenvalues. Physically, these constraints stem from the limitation of the feedback strength or finite Hamiltonian resources. Here, following Ref. 31 by Doherty et al., we adopt the last type of constraint, namely, $\operatorname{Tr}\left[H_{f b}^{2}\right]$ $\leqslant \mu$. Under this constraint, the feedback Hamiltonian can be constructed in terms of

$$
H_{f b}=i \lambda\left[\left|\psi_{d}(t)\right\rangle\left\langle\psi_{d}(t)\right|, \rho_{c}(t)\right],
$$

where $\lambda=\sqrt{\mu / 2\left(a-b^{2}\right)}$, with $a=\left\langle\psi_{d}(t)\left|\rho_{c}(t)^{2}\right| \psi_{d}(t)\right\rangle$ and $b$ $=\left\langle\psi_{d}(t)\left|\rho_{c}(t)\right| \psi_{d}(t)\right\rangle$.

Combining the above feedback Hamiltonian with the previously developed state unravelling scheme, the estimated state $\rho_{c}(t)$ can be straightforwardly calculated, leading to the state propagation in the presence of feedback. Figure 3 shows the control result with quantum feedback, where the ensemble average has been made over a large number of Monte Carlo simulated trajectories. Here the measurement voltage is quite moderate, i.e., $V=3 \Omega$, which is beyond the theoretical description in the large voltage limit as previously studied. ${ }^{13,15,17-20}$ We observed that the control effect is evident: by increasing the feedback strength $\lambda$, the measurement induced back-action can be largely eliminated and the desired coherent oscillation of the qubit can be maintained for arbitrarily long time.

It will be of interest to compare the quantum measurement in the presence of feedback to the well-known quantum nondemolition (QND) measurement. For the solid-state qubit, elegant schemes of QND measurement have been proposed very recently. ${ }^{33,34}$ Here, for the qubit measurement under consideration, we demonstrate that the QND

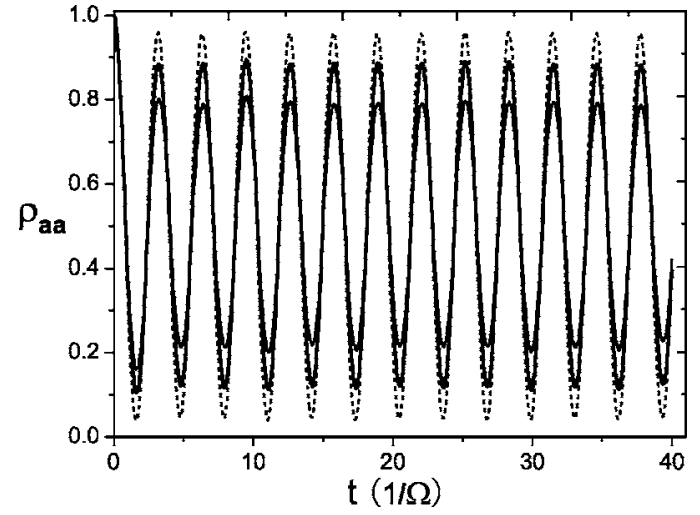

FIG. 3. Feedback control of the coherent oscillation of qubit state, resulting from feedback strengths $\lambda=0.5$ (thin solid line), 1.0 (thick solid line), and 3.5 (dashed line). The parameters are $\mathcal{T}$ $=20.0 \Omega, \quad \chi=0.7 \Omega, \quad V=3.0 \Omega, \quad \epsilon=0.0 \Omega, \quad T=1.0 \Omega$, and $g_{L(R)}$ $=1 / \sqrt{2 \pi} \Omega$.

measurement is equivalent to the usual back-action-present measurement plus quantum feedback. In Fig. 4 we present the calculated output power spectrum of the QPC detector. The peak at $\omega=\Omega$ indicates the coherent oscillation of the qubit. In the absence of feedback, it has been shown that the peak-to-background ratio cannot be larger than $4,{ }^{16}$ due to the back-action of measurement. In the presence of feedback, however, we obtain a very sharp peak here, which indicates almost ideal coherent oscillations.

Theoretically, since no steady state is available in the presence of feedback, the start time of the qubit evolution is chosen as the initial time of the current correlation function, and the noise spectrum is the Fourier transform of the correlation function with respect to a later time (difference). Experimentally, this feedback-induced sharper peak can be employed as an indicator for the feedback effect in practice. We expect that this kind of experiment can be performed in the near future.

Finally, we address the effect of measurement voltage. Figure 5 shows the synchronization degree of the feedback

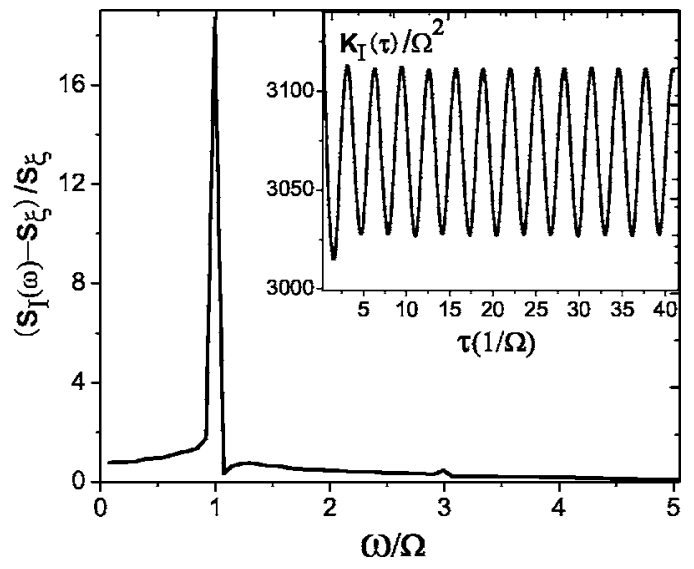

FIG. 4. Narrowed coherence peak in the noise spectrum as an indicator for the feedback effect (inset: the coherently oscillating correlation function). The parameters are $\mathcal{T}=10.0 \Omega, \chi=0.2 \Omega, V$ $=3.0 \Omega, \epsilon=0.0 \Omega, T=0.5 \Omega, \lambda=15$, and $g_{L(R)}=1 / \sqrt{2 \pi} \Omega$. 


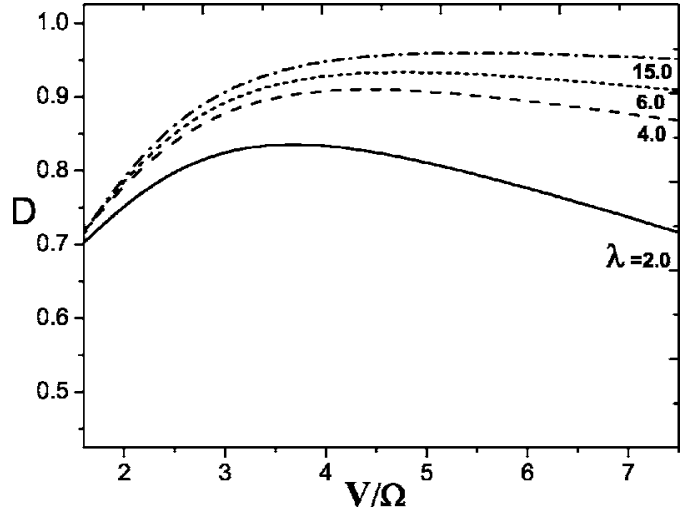

FIG. 5. Effect of measurement voltage on the feedback control. Here the quantity synchronization degree, which is defined as $D$ $=2\left\langle\operatorname{Tr}\left[\rho_{c}(t) \rho_{d}(t)\right]\right\rangle-1$, with $\langle\cdots\rangle$ meaning the average over time, is employed to characterize the control quality. Note that for perfect control the synchronization degree is unity (i.e., $D=1$ ). The parameters are $\mathcal{T}=20.0 \Omega, \chi=0.7 \Omega, \epsilon=0.0 \Omega, T=1.0 \Omega$, and $g_{L(R)}$ $=1 / \sqrt{2 \pi} \Omega$.

versus the measurement voltage. It is of interest to note that there exists an optimal measurement voltage for a relatively small feedback strength. This is because for larger voltage the back-action is relatively too strong, while for smaller voltage the information of the measured state cannot be extracted out efficiently. As a result, the turnover behavior of the synchronization degree versus voltage is found. However, with the increase of the feedback strength, the strong back-action can be eliminated more efficiently by the feedback. In this case, the synchronization degree does not decrease considerably as the measurement voltage increases, as shown in Fig. 5.

\section{CONCLUDING REMARKS}

To summarize, in the context of continuous quantum measurement and coherence control of solid-state charge qubit, we have presented an unravelling scheme for the nonLindblad-type master equation. Based on it, we also constructed an efficient method to calculate the noise spectrum, which can be regarded as a generalization of the standard quantum-jump theory developed in quantum optics. Despite the absence of analytic formalism, the numerical implementation in practice was demonstrated to be straightforward and efficient. An illustrative application was further contributed to the quantum feedback control under arbitrary measurement voltages. The detector noise spectrum under feedback was calculated, and narrowing it clearly reflected the control effect. Also, the effect of measurement voltage was discussed.

The present study has been focused on the setup of a double-dot qubit measured by quantum point contact. However, for other solid-state setup such as a charge qubit measured by a single-electron transistor, similar unravelling scheme can be constructed. Owing to the fact that, in general (e.g., in the presence of many-body Coulomb correlations), the measurement dynamics is not governed by a Lindblad- type master equation, the present $n$-resolved master equation based unravelling scheme seems quite desirable. Finally, in spite of the various unravelling schemes for a non-Lindbladtype master equation or even for non-Markovian dissipative systems, to our knowledge, all of them are largely mathematical. Therefore, the present physical unravelling scheme is of interest and valuable, which may find applications in the field of solid-state quantum information.

\section{ACKNOWLEDGMENTS}

This work was supported by the National Natural Science Foundation of China under Grant Nos. 60425412 and 90503013, and the Major State Basic Research Project of China under Grant No. G001CB3095.

\section{APPENDIX A: MATRIX ELEMENTS OF $\mathcal{M}(k)$}

The coefficients in Eq. (5) read

$$
\begin{gathered}
a_{1}=-Q_{a a}\left[\widetilde{Q}_{a a}-e^{i k} \widetilde{Q}_{a a}^{(-)}-e^{-i k} \widetilde{Q}_{a a}^{(+)}\right] \\
a_{2}=-\frac{Q_{a a}}{2}\left[\widetilde{Q}_{a b}-e^{i k} \widetilde{Q}_{a b}^{(-)}-e^{-i k} \widetilde{Q}_{a b}^{(+)}\right] \\
b_{1}=-Q_{b b}\left[\widetilde{Q}_{b b}-e^{i k} \widetilde{Q}_{b b}^{(-)}-e^{-i k} \widetilde{Q}_{b b}^{(+)}\right] \\
b_{2}=-\frac{Q_{b b}}{2}\left[\widetilde{Q}_{b a}-e^{i k} \widetilde{Q}_{b a}^{(-)}-e^{-i k} \widetilde{Q}_{b a}^{(+)}\right] \\
c_{1}=-\frac{1}{2}\left[\left(Q_{a a} \widetilde{Q}_{a a}+Q_{b b} \widetilde{Q}_{b b}\right)-e^{i k}\left(Q_{a a} \widetilde{Q}_{b b}^{(-)}+Q_{b b} \widetilde{Q}_{a a}^{(-)}\right)\right. \\
\left.-e^{-i k}\left(Q_{a a} \widetilde{Q}_{b b}^{(+)}+Q_{b b} \widetilde{Q}_{a a}^{(+)}\right)\right] \\
c_{2}=-\frac{1}{2}\left[Q_{a a} \tilde{Q}_{a b}-e^{i k} Q_{b b} \widetilde{Q}_{a b}^{(-)}-e^{-i k} Q_{b b} \widetilde{Q}_{a b}^{(+)}\right] \\
c_{3}=-\frac{1}{2}\left[Q_{b b} \widetilde{Q}_{b a}-e^{i k} Q_{a a} \widetilde{Q}_{b a}^{(-)}-e^{-i k} Q_{a a} \widetilde{Q}_{b a}^{(+)}\right]
\end{gathered}
$$

where

$$
\begin{gathered}
\widetilde{Q}_{a a}^{( \pm)}=\widetilde{C}^{( \pm)}(0)\left[T+\frac{1}{2} \chi\left(1+\cos ^{2} \theta\right)\right]+\chi \lambda_{ \pm} \sin ^{2} \theta, \\
\widetilde{Q}_{b b}^{( \pm)}=\widetilde{C}^{( \pm)}(0)\left[T+\frac{1}{2} \chi\left(1+\sin ^{2} \theta\right)\right]-\chi \lambda_{ \pm} \sin ^{2} \theta, \\
\widetilde{Q}_{a b}^{( \pm)}=\frac{1}{2} \chi \widetilde{C}^{( \pm)}(0) \sin \theta \cos \theta+\chi \sin \theta\left(\bar{\lambda}_{ \pm}-\lambda_{ \pm} \cos \theta\right), \\
\widetilde{Q}_{b a}^{( \pm)}=\frac{1}{2} \chi \widetilde{C}^{( \pm)}(0) \sin \theta \cos \theta-\chi \sin \theta\left(\bar{\lambda}_{ \pm}+\lambda_{ \pm} \cos \theta\right), \\
Q_{a a}=T+\chi, \quad Q_{b b}=T, \quad Q_{a b}=Q_{b a}=0,
\end{gathered}
$$

and 


$$
\begin{aligned}
& \lambda_{ \pm}=\frac{1}{4}\left[\tilde{C}^{( \pm)}(-\Delta)+\tilde{C}^{( \pm)}(\Delta)\right], \\
& \bar{\lambda}_{ \pm}=\frac{1}{4}\left[\tilde{C}^{( \pm)}(-\Delta)-\tilde{C}^{( \pm)}(\Delta)\right] .
\end{aligned}
$$

\section{APPENDIX B: NOISE SPECTRUM}

In this Appendix, along the line of the conventional quantum-jump theory, we extend the method of noise spectrum calculation to the unravelling approach developed in this work. Consider the correlation function $E[d n(t$ $+\tau) d n(t)]$, where $E[d n(t)]=\Sigma_{n_{1}} n_{1} \operatorname{Tr}\left[\mathcal{U}\left(n_{1}, d t\right) \rho(t)\right]$.

First, for the case $\tau>0$,

$$
\begin{aligned}
E[d n(t+\tau) d n(t)]= & \sum_{n_{1}} n_{1} \operatorname{Prob}\left[d n(t)=n_{1}\right] \\
& \times E\left[\left.d n_{c}(t+\tau)\right|_{d n(t)=n_{1}}\right],
\end{aligned}
$$

where $\operatorname{Prob}\left[d n(t)=n_{1}\right]=\operatorname{Tr}\left[\mathcal{U}\left(n_{1}, d t\right) \rho(t)\right]$. At time $t+d t$, a definite value of $n_{1}$ is picked out and the qubit state undergoes an immediate collapse, i.e., $\rho_{c}(t+d t)$ $=\mathcal{U}\left(n_{1}, d t\right) \rho(t) / \operatorname{Prob}\left[d n(t)=n_{1}\right]$.

During the time period $[t+d t, t+\tau]$, one can ignore the measurement records owing to the ensemble nature of the correlation function, therefore

$$
\begin{aligned}
& E\left[\left.d n_{c}(t+\tau)\right|_{d n(t)=n_{1}}\right] \\
& \quad=\sum_{n_{2}} n_{2} \operatorname{Tr}\left\{\mathcal{U}\left(n_{2}, d t\right) e^{\mathcal{L}(\tau-d t)} \frac{\mathcal{U}\left(n_{1}, d t\right) \rho(t)}{\operatorname{Prob}\left[d n(t)=n_{1}\right]}\right\} .
\end{aligned}
$$

To the leading order of $d t$ we have

$$
\begin{aligned}
& E[d n(t+\tau) d n(t)] \\
& =\sum_{n_{1} n_{2}} n_{2} \operatorname{Tr}\left\{\mathcal{U}\left(n_{2}, d t\right) e^{\mathcal{L} \tau} \frac{\mathcal{U}\left(n_{1}, d t\right) \rho(t)}{\operatorname{Tr}\left[\mathcal{U}\left(n_{1}, d t\right) \rho(t)\right]}\right\} \\
& \quad \times n_{1} \operatorname{Tr}\left[\mathcal{U}\left(n_{1}, d t\right) \rho(t)\right]=\operatorname{Tr}\left[\overline{\mathcal{U}} e^{\mathcal{L} \tau} \overline{\mathcal{U}} \rho(t)\right] d t^{2} .
\end{aligned}
$$

Here we have introduced $\overline{\mathcal{U}} \equiv \Sigma_{n} n \mathcal{U}(n, d t) / d t$.

Next for $\tau=0$ we have

$$
\begin{aligned}
E\left[d n(t)^{2}\right] & =\sum_{n} n^{2} \operatorname{Prob}[d n(t)=n]=\sum_{n} n^{2} \operatorname{Tr}[\mathcal{U}(n, d t) \rho(t)] \\
& =\operatorname{Tr}\left[\overline{\mathcal{U}}^{\prime} \rho(t)\right] d t
\end{aligned}
$$

where $\overline{\mathcal{U}}^{\prime}=\sum_{n} n^{2} \mathcal{U}(n, d t) / d t$. For short time $\tau$, this equal-time correlation will be dominant, and $E\left[\frac{d n(t+\tau)}{d t} \frac{d n(t)}{d t}\right]$ can be treated as $\delta$-correlated noise for a suitably defined $\delta$ function. We thus obtain

$$
\begin{aligned}
K_{I}(\tau)= & E\left[\frac{d n(t+\tau)}{d t} \frac{d n(t)}{d t}\right]-E\left[\frac{d n(t+\tau)}{d t}\right] E\left[\frac{d n(t)}{d t}\right] \\
= & \operatorname{Tr}\left[\overline{\mathcal{U}} e^{\mathcal{L} \tau} \overline{\mathcal{U}} \rho(t)\right]-\operatorname{Tr}[\overline{\mathcal{U}} \rho(t+\tau)] \operatorname{Tr}[\overline{\mathcal{U}} \rho(t)] \\
& +\operatorname{Tr}\left[\overline{\mathcal{U}}^{\prime} \rho(t)\right] \delta(\tau) .
\end{aligned}
$$

Finally, it should be noted that $[d n(t)]^{2} \neq d n(t)$ in our above treatment. This differs from the conventional quantum-jump theory where the stochastic number $d n(t)=0$ or 1 in the point process.
*Present address: Department of Chemistry, Hong Kong University of Science and Technology, Kowloon, Hong Kong.

†lectronic address: xqli@red.semi.ac.cn

${ }^{1}$ N. Gisin, Phys. Rev. Lett. 52, 1657 (1984).

${ }^{2}$ P. Zoller, M. Marte, and D. F. Walls, Phys. Rev. A 35, 198 (1987).

${ }^{3}$ N. Gisin and I. C. Percival, J. Phys. A 25, 5677 (1992).

${ }^{4}$ J. Dalibard, Y. Castin, and K. Molmer, Phys. Rev. Lett. 68, 580 (1992).

${ }^{5}$ H. J. Carmichael, An Open System Approach to Quantum Optics, Lecture Notes in Physics (Springer, Berlin, 1993).

${ }^{6}$ H. M. Wiseman and G. J. Milburn, Phys. Rev. A 47, 1652 (1993).

${ }^{7}$ M. B. Plenio and P. L. Knight, Rev. Mod. Phys. 70, 101 (1998).

${ }^{8}$ H-P. Breuer, B. Kappler, and F. Petruccione, Phys. Rev. A 59, 1633 (1999).

${ }^{9}$ A. Imamoglu, Phys. Rev. A 50, 3650 (1994).

${ }^{10}$ W. T. Strunz, L. Diósi, and N. Gisin, Phys. Rev. Lett. 82, 1801 (1999); W. T. Strunz, L. Diósi, N. Gisin, and T. Yu, ibid. 83, 4909 (1999).

${ }^{11}$ J. T. Stockburger and C. H. Mak, Phys. Rev. Lett. 80, 2657
(1998); J. Chem. Phys. 110, 4983 (1999); J. T. Stockburger and H. Grabert, Chem. Phys. 268, 249 (2001); Phys. Rev. Lett. 88, 170407 (2002).

${ }^{12}$ J. Shao, J. Chem. Phys. 120, 5053 (2004).

${ }^{13}$ S. A. Gurvitz, Phys. Rev. B 56, 15215 (1997).

${ }^{14}$ A. Shnirman and G. Schön, Phys. Rev. B 57, 15400 (1998); Y. Makhlin, G. Schön, and A. Shnirman, Rev. Mod. Phys. 73, 357 (2001).

${ }^{15}$ A. N. Korotkov, Phys. Rev. B 60, 5737 (1999); 63, 115403 (2001).

${ }^{16}$ A. N. Korotkov and D. V. Averin, Phys. Rev. B 64, 165310 (2001).

${ }^{17}$ H. S. Goan, G. J. Milburn, H. M. Wiseman, and H. B. Sun, Phys. Rev. B 63, 125326 (2001).

${ }^{18}$ R. Ruskov and A. N. Korotkov, Phys. Rev. B 66, 041401(R) (2002); A. N. Korotkov, ibid. 71, 201305(R) (2005); Q. Zhang, R. Ruskov, and A. N. Korotkov, ibid. 72, 245322 (2005).

${ }^{19}$ A. Hopkins, K. Jacobs, S. Habib, and K. Schwab, Phys. Rev. B 68, 235328 (2003).

${ }^{20}$ R. Ruskov, K. Schwab, and A. N. Korotkov, IEEE Trans. Nano- 
technol. 4, 132 (1995); Phys. Rev. B 71, 235407 (2005).

${ }^{21}$ T. Hayashi, T. Fujisawa, H. D. Cheong, Y. H. Jeong, and Y. Hirayama, Phys. Rev. Lett. 91, 226804 (2003); J. M. Elzerman, R. Hanson, J. S. Greidanus, L. H. Willems van Beveren, S. De Franceschi, L. M. K. Vandersypen, S. Tarucha, and L. P. Kouwenhoven Phys. Rev. B 67, 161308(R) (2003); J. R. Petta, A. C. Johnson, C. M. Marcus, M. P. Hanson, and A. C. Gossard, Phys. Rev. Lett. 93, 186802 (2004).

${ }^{22}$ Xin-Qi Li, Wen-Kai Zhang, Ping Cui, Jiushu Shao, Zhongshui Ma, and YiJing Yan, Phys. Rev. B 69, 085315 (2004).

${ }^{23}$ Xin-Qi Li, Ping Cui, and YiJing Yan, Phys. Rev. Lett. 94, 066803 (2005).

${ }^{24}$ Hsi-Sheng Goan and Gerard J. Milburn, Phys. Rev. B 64, 235307 (2001); Hsi-Sheng Goan, Quantum Inf. Comput. 3, 121 (2003).

${ }^{25}$ A. O. Caldeira and A. J. Leggett, Ann. Phys. (N.Y.) 149, 374 (1983).

${ }^{26}$ H. M. Wiseman and G. J. Milburn, Phys. Rev. Lett. 70, 548 (1993); Phys. Rev. A 47, 642 (1993); 49, 4110 (1994).

${ }^{27}$ H. M. Wiseman, Phys. Rev. Lett. 75, 4587 (1995); H. M. Wise- man and R. B. Killip, Phys. Rev. A 57, 2169 (1998); M. A. Armen, J. K. Au, J. K. Stockton, A. C. Doherty, and H. Mabuchi, Phys. Rev. Lett. 89, 133602 (2002).

${ }^{28}$ T. C. Ralph, A. P. Lund, and H. M. Wiseman, J. Opt. B: Quantum Semiclassical Opt. 7, S245 (2005).

${ }^{29}$ N. Katz, M. Ansmann, R. C. Bialczak, E. Lucero, R. McDermott, M. Neeley, M. Steffen, E. M. Weig, A. N. Cleland, J. M. Martinis, and A. N. Korotkov, Science 312, 1498 (2006).

${ }^{30}$ A. C. Doherty and K. Jacobs, Phys. Rev. A 60, 2700 (1999); H. M. Wiseman, S. Mancini, and Jin Wang, ibid. 66, 013807 (2002).

${ }^{31}$ A. C. Doherty, K. Jacobs, and G. Jungman, Phys. Rev. A 63, 062306 (2001).

${ }^{32}$ Jinshuang Jin, Xin-Qi Li, and YiJing Yan, Phys. Rev. B 73, 233302 (2006).

${ }^{33}$ D. V. Averin, Phys. Rev. Lett. 88, 207901 (2002).

${ }^{34}$ Andrew N. Jordan and Markus Buttiker, Phys. Rev. B 71, 125333 (2005). 